\title{
The Commissioning of the CMS Electromagnetic Calorimeter Light Monitoring System
}

\author{
Toyoko J. Orimoto, \\ On behalf of the CMS ECAL Group
}

\begin{abstract}
The Compact Muon Solenoid (CMS) Experiment, located at the Large Hadron Collider at CERN, is a general purpose particle detector experiment with a high resolution electromagnetic calorimeter (ECAL). I report on the commissioning of the light monitoring system, which tracks changes in transparency of the lead tungstate crystals and is thus crucial for maintaining the energy resolution of the calorimeter.
\end{abstract}

Index Terms-LHC, CMS, electromagnetic calorimeter, crystal calorimeter, light monitoring

\section{INTRODUCTION}

$\mathbf{U}$ NDERSTANDING the origins of electroweak symmetry breaking, and in particular discovering the Higgs boson predicted by the Standard Model, is the main motivation for building the Compact Muon Solenoid (CMS) experiment at the Large Hadron Collider (LHC) at CERN.

The CMS electromagnetic calorimeter (ECAL) was designed to optimize the resolution for the discovery of the Higgs in the distinctive, yet challenging, diphoton channel. In the low Higgs mass range around $120 \mathrm{GeV}$, the diphoton channel will be the most promising for discovery because of its distinctive signature. In this mass range, the decay width of the Higgs is expected to be very narrow, and the discovery potential is directly related to the reconstructed mass width, or rather, the energy and position resolution of the electromagnetic calorimeter.

The CMS ECAL is a high-resolution, high-granularity scintillating crystal calorimeter, consisting of 75,848 lead-tungstate $\left(\mathrm{PbWO}_{4}\right)$ crystals [1],[2]. $\mathrm{PbWO}_{4}$ crystals were chosen because of their short radiation length, small Molière radius, and fast speed as a scintillator. The design energy resolution of the ECAL has a constant term of $0.5 \%$, and to maintain this constant term, in situ calibration and monitoring of the crystals must be performed. Exposure at the level of the nominal LHC luminosity causes a decrease in crystal transparency due to radiation induced absorption. The changes in transparency are monitored with a high precision, laser-based light monitoring system, the performance of which is described here.

\section{RADIATION INDUCED CRYSTAL TRANSPARENCY CHANGE}

At the LHC design luminosity, the CMS detector will be exposed to a very harsh radiation environment. The $\mathrm{PbWO}_{4}$ crystals are radiation hard up to a high integrated dosage, but

T. J. Orimoto is with the California Institute of Technology, Pasadena, CA 91125, USA (e-mail: toyoko@hep.caltech.edu). nonetheless suffer from dose-rate dependent radiation damage, resulting in a decrease in crystal transparency.

Previous studies have shown that the scintillation mechanism of $\mathrm{PbWO}_{4}$ crystals is not affected by radiation, and the loss of light output is due only to the absorption caused by radiation induced color centers [3]. The time scale of the transparency change is fast, on the order of a few hours, compared to the time scale of days, weeks, or months needed for calibration with physics events to reach sub-percent precision. During irradiation, the annihilation and creation of color centers reach an equilibrium, depending on the applied dose rate. Although the crystals will self-recover in absence of radiation, this recovery takes place on the order of a week, while the time cycle of the LHC operation is 12 hours. Therefore, changes in crystal transparency, and therefore calorimeter response, due to radiation damage must be corrected for to maintain the energy resolution of the detector.

\section{THE LASER MONITORING SYSTEM}

The CMS ECAL utilizes a laser monitoring system to monitor the light output of the crystals. With this system, we can measure the change in transparency of each crystal continuously during LHC operation, with very high precision. In order to maintain the constant term in the ECAL resolution to $0.5 \%$, the measurement of the transparency change must be made with an accuracy of greater than $0.2 \%$. The ECAL readout electronics require that the laser pulse width be shorter than $40 \mathrm{~ns}$ and stable at a level better than $10 \%$. Sychronization with the LHC clock requires that the laser pulse timing be stable to a few ns. In addition, the pulse energy must be on the order of $1 \mathrm{~mJ} /$ pulse, which is equivalent to $1.3 \mathrm{TeV}$ in the dynamic range of the system, to provide sufficient light to monitor the entire ECAL in approximately 20 minutes.

To meet these requirements, a dual-stage, laser system has been designed, constructed and commissioned by the Caltech and Saclay groups [4]. The monitoring light source consists of two sets of Nd:YLF laser pumped Ti:Sapphire lasers, which provide two wavelengths, available using a $3 \times 1$ optical switch. The selected wavelength is then sent to each ECAL monitoring element using a $1 \times 88$ optical switch.

In 2006, a software feedback system was implemented so that the long term stability of the laser pulse intensity and FWHM width are maintained at the level of a few percent, with a laser pulse timing jitter of less than $2 n s$. The long term stability was observed in more than 2,000 hours of test beam data, as shown in Fig. 1. With an integrating sphere based light 


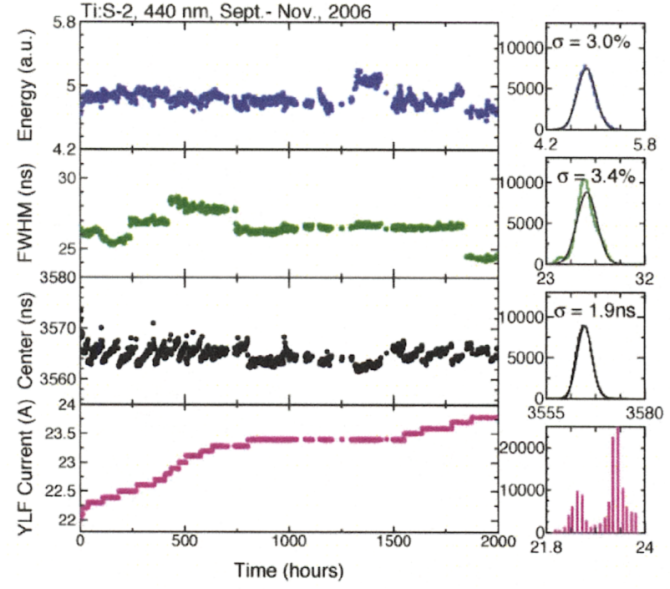

Fig. 1: Stability of the laser monitoring system, as measured in test beam.

distribution system, the stability of the normalized monitoring response is about $0.1 \%$. With such performance, even small changes in transparency can be monitored with precision.

Typical variations in crystal transparency measured by using blue laser pulses are at the level of a few percent. The corresponding variations in the crystal light output, measured by using an electron beam, can be as large as $5 \%$ in the ECAL barrel and even greater in the endcaps for radiation levels at LHC design luminosity.

\section{Performance Results from Test Beams}

The performance of the monitoring system and the transparency correction were tested during dedicated irradiation runs at test beams in 2006 and 2007 [5]. These dedicated studies were performed by emulating the dose rates expected at the LHC by irradiating the crystals with an electron beam at high rates. During the irradiation periods, the crystals were regularly monitored with laser runs. Fig. 2a shows the changes in crystal transparency during the damage and recovery cycles in test beam data.

Prior studies have shown that the decrease in scintillation signal response due to a particle traversing a crystal is directly proportional to the decrease in the injected laser light monitoring signal. The loss in the response of the crystals to electron beam runs can be correlated with the loss in response to laser runs, by the equation, $E(t) / E\left(t_{0}\right)=\left[L(t) / L\left(t_{0}\right)\right]^{\alpha}$, where $E(t) / E\left(t_{0}\right)$ corresponds to the normalized electron beam response, $L(t) / L\left(t_{0}\right)$ is the normalized response to laser runs, and $\alpha$ is the correlation constant which relates these two responses. Fig. $2 b$ shows the fit for $\alpha$ for one of the ECAL barrel crystals, from test beam data. The spread of $\alpha$ values, as shown in Fig. 2c, has been measured to be around $5 \%$ in a sample of 28 crystals. Such a small dispersion will allow us to use the same $\alpha$ parameter for all ECAL crystals during LHC
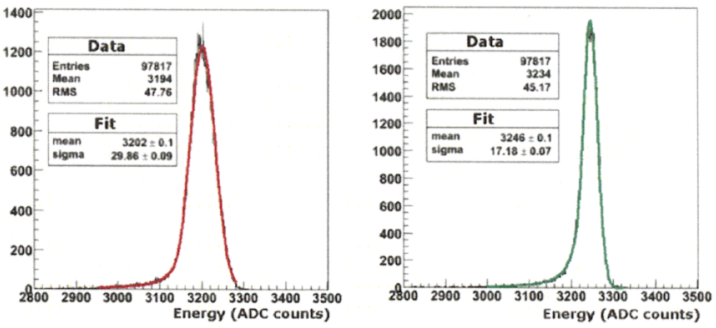

Fig. 3: Energy resolution of an ECAL barrel crystal, before (left) and after (right) applying the transparency correction determined in Fig. 2.

operation. As shown in Figure 3, the energy resolution before and after the monitoring corrections, for $120 \mathrm{GeV}$ electrons, reconstructed by summing the energy in $3 \times 3$ crystals, is $0.93 \%$ and $0.53 \%$ as measured with test beam data.

\section{LASER Monitoring Dataflow}

During LHC running, laser data are collected during "abort gap" events, periods in absence of collisions lasting for $3 \mu \mathrm{s}$ every $90 \mu$ s. Fig. 4 shows a diagram of the laser data flow, during in situ operation of the monitoring system. The abort gap events, containing laser data amongst other data, are sent to a laser PC farm, at which point the events are sorted and analyzed to extract data relevant for the transparency correction. The data are inserted into the "online" database, located at CMS Point 5, and then transferred to the "offline" database, during which any necessary corrections are applied to the laser data. During the offline reconstruction of CMS data, the values in the database are used to compute and apply the transparency correction to physics events.

\section{COMMISSIONING OF THE LASER MONITORING SYSTEM AND FIRST LHC DATA}

Two laser systems (blue/green and IR/red) have been installed and commissioned in the CMS underground cavern at Point 5, and first laser data has been collected. During the commissioning phase of CMS and first LHC beam, we have not reached radiation levels which would induce transparency changes in the calorimeter crystals. However, the laser monitoring system has been used for the commissioning of ECAL. For instance, the laser system provides a precise way

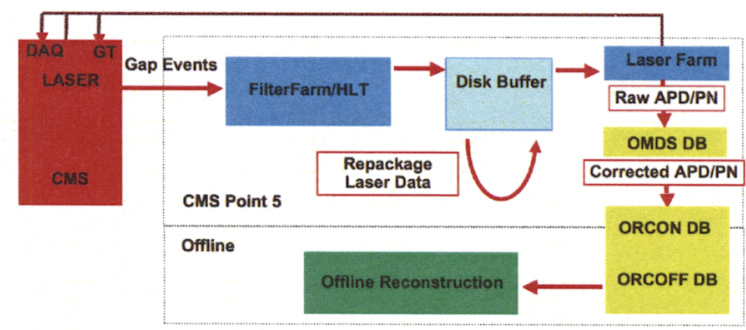

Fig. 4: Schematic diagram of the laser monitoring data flow from the CMS detector to offline reconstruction. 


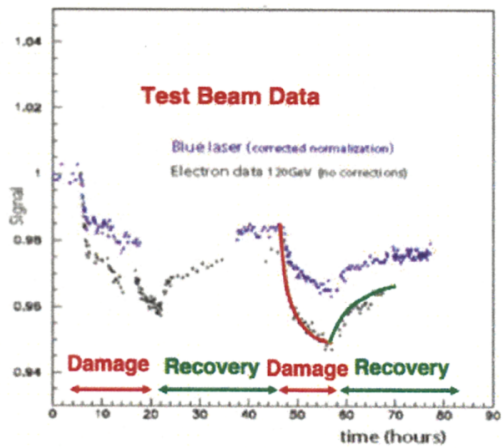

(a)

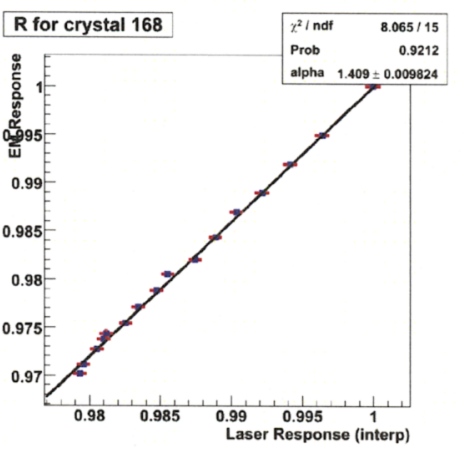

(b)

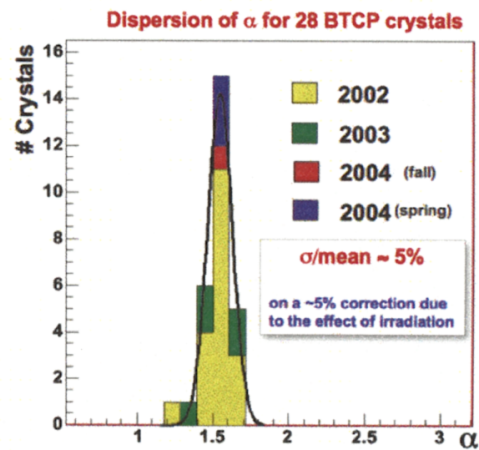

(c)

Fig. 2: (a) The changes in responses to electron beam and laser runs during the damage and recovery cycles of the crystals, as seen in test beam data. (b) The correlation between the responses to electron and laser test beam data, for one of the ECAL barrel crystals. (c) The distribution of $\alpha$ values for 28 different ECAL crystals.

to evaluate the timing of the ECAL signal. Utilizing laser data and first LHC beam data, we have adjusted for differences in timing between different ECAL readout units. In addition, the laser data has confirmed that the timing of all ECAL readout units is within 2 ns of the mean value.

\section{CONCLUSION}

The CMS ECAL laser monitoring system has been tested at test beams at CERN, and commissioned at CMS Point 5 underground. The laser monitoring system will provide corrections for light output fluctuations caused by variations in crystal transparency during the radiation damage and recovery process. The stability of the system has been exhibited to be on the order of $0.1 \%$. With such performance, even small changes in transparency can be monitored with precision.

\section{ACKNOWLEDGMENT}

I would like to acknowledge the CMS ECAL Collaboration for their effort and dedication. I would also like to thank my colleagues from the laser monitoring group, particularly $\mathrm{A}$. Bornheim and R.Y. Zhu, for their input and support.

\section{REFERENCES}

[1] “The Electromagnetic Calorimeter Technical Design Report", CMS Collaboration, CERN/LHCC 97-33, 1997.

[2] R. Adolphi et al. [CMS Collaboration], "The CMS experiment at the CERN LHC," JINST 3. S08004 (2008).

[3] "Radiation Induced Color Centers and Light Monitoring for Lead Tungstate Crystals", X. Qu, L. Zhang, R.Y. Zhu, IEEE Trans. Nucl. Sci. NS-47 (2000).

[4] "Performance of the Monitoring Light Source for the CMS Lead Tungstate Crystal Calorimeter", IEEE Trans. Nucl. Sci. Vol. 52 No. 4 (2005) 1123-1130.

[5] "ECAL Monitoring Light Source at H4", D. Bailleux, A. Bornheim, L.Y Zhang, K.J. Zhu, R.Y. Zhu, and D. Liu, CMS IN 2003/045. 